\title{
Response to letter to the editor of Carcinogenesis by Li et al., 2020
}

Kountouras, Jannis ; Papaefthymiou, Apostolis ; Polyzos, Stergios A ; Zavos, Christos ; Doulberis, Michael

DOI: https://doi.org/10.1093/carcin/bgaa042

Posted at the Zurich Open Repository and Archive, University of Zurich

ZORA URL: https://doi.org/10.5167/uzh-189315

Journal Article

Accepted Version

Originally published at:

Kountouras, Jannis; Papaefthymiou, Apostolis; Polyzos, Stergios A; Zavos, Christos; Doulberis, Michael (2020). Response to letter to the editor of Carcinogenesis by Li et al., 2020. Carcinogenesis, 41(4):539-540.

DOI: https://doi.org/10.1093/carcin/bgaa042 


\section{Carcinogenesis}

\section{Letter to the editor re: Li et al. (2020), 'The potential role of bacteria in pancreatic cancer: A systematic review'}

\begin{tabular}{|r|l|}
\hline Journal: & Carcinogenesis \\
\hline Manuscript ID & CARCIN-2020-00075 \\
\hline Danuscript Type: & Letter to the Editor \\
\hline Author: & 16 -Feb-2020 \\
\hline Complete List of Authors: & $\begin{array}{l}\text { Kountouras, Jannis; Aristotle University of Thessaloniki, Department of } \\
\text { Medicine, Second Medical Clinic, Ippokration Hospital } \\
\text { Papaefthymiou, Apostolis; Aristotle University of Thessaloniki, } \\
\text { Department of Medicine, Second Medical Clinic, Ippokration Hospital } \\
\text { Polyzos, Stergios; Aristotle University of Thessaloniki, Department of } \\
\text { Medicine, Second Medical Clinic, Ippokration Hospital } \\
\text { Zavos, Christos; Aristotle University of Thessaloniki, Department of } \\
\text { Medicine, Second Medical Clinic, Ippokration Hospital } \\
\text { Doulberis, Michael; Aristotle University of Thessaloniki, Laboratory of } \\
\text { Pathology, Faculty of Health Sciences, School of Veterinary Medicine }\end{array}$ \\
\hline Keywords: & $\begin{array}{l}\text { Helicobacter pylori; autoimmune pancreatitis; immunoglobulin G4- } \\
\text { related disease; pancreatic cancer; apoptosis }\end{array}$ \\
\hline & \\
\hline
\end{tabular}




\title{
Letter to the editor re: Li et al. (2020), 'The potential role of bacteria in pancreatic cancer: A systematic review'
}

\author{
Jannis Kountouras ${ }^{1,{ }^{*}}$, Apostolis Papaefthymiou ${ }^{1}$, Stergios A. Polyzos ${ }^{1,2}$ \\ Christos Zavos ${ }^{1}$, Michael Doulberis ${ }^{1,3}$ \\ 1Department of Medicine, Second Medical Clinic, Aristotle University of \\ Thessaloniki, Ippokration Hospital, Thessaloniki 54642, Macedonia, Greece \\ 2First Department of Pharmacology, Faculty of Medicine, Aristotle University \\ of Thessaloniki, Thessaloniki 54642, Macedonia, Greece \\ ${ }^{3}$ Department of Gastroenterology and Hepatology, University of Zurich, Zurich \\ 8091, Switzerland
}

Key words: Helicobacter pylori; autoimmune pancreatitis; immunoglobulin G4related disease; pancreatic cancer; apoptosis Financial support: None

Potential competing interests: None

To whom correspondence should be addressed: Jannis Kountouras, MD, PhD

Professor of Medicine 8 Fanariou St, Byzantio 551 33, Thessaloniki, Macedonia Greece Tel: +30-2310-892238, Fax: +30-2310-992794 E-mail: jannis@auth.gr,ancoratus2010@gmail.com 
Dear Sir,

In their review $\mathrm{Li}$ et al. [1] focused on bacteria and particularly on Helicobacter pylori infection $(\mathrm{Hp}-\mathrm{I})$ involvement in pancreatic cancer pathophysiology by many molecular potential mechanisms.

$\mathrm{Hp}$ is the fundamental cause of gastric ulcer disease also associated with autoimmune pancreatitis (AIP) [2] and as a class I carcinogen may also be implicated, through molecular mimicry, in the pathophysiology of AIP and probably of pancreatic malignancy; this pathogen appears to be involved in AIP via induction of autoimmunity and apoptosis [3], and, apart from other molecular mechanisms some of which also mentioned by the authors $[1,4]$ $\mathrm{Hp}$-I may also contribute to pancreatic carcinogenesis by altered apoptotic pathways $[4,5]$.

Specifically, relative data [6] identified that: a) peptide AIP1-7 showed homology with Hp plasminogen-binding protein (PBP) and with the enzyme biquitin-protein ligase E3 component n-recognin 2, expressed in pancreatic acinar cells; and b) the antibody against PBP was associated with AIP. Hp was found to bind plasminogen by $H p$ PBPs, enhancing its virulence [7]. These data appear to support our proposition $[3,8]$ that $H p$ might trigger AIP through molecular mimicry and its increased virulence. Plasminogen binding and its conversion to plasmin is the only proteolytic activity of $H p$, it may enhance tissue damage [7] and is involved in carcinogenesis; anti-PBP peptide antibody is present in patients with pancreatic cancer [6]. Besides, Guarneri et al. [9] found homology between human pancreatic autoantigen carbonic anhydrase II and a-carbonic anhydrase of $\mathrm{Hp}$, a fundamental 
enzyme for Hp survival and proliferation; the homologous segments contained the binding motif of the HLA molecule DRB1*0405 [9]. These data further strengthen our assumption that $\mathrm{Hp}$ infection can trigger AIP in genetically predisposed subjects and consequence pancreatic cancer.

In this regard several studies have proposed that the risk of pancreatic malignancy is high in patients with AIP [10]; IgG4-related disease is associated with cancer development especially during the first year after diagnosis, thereby signifying that active IgG4-related disease appears to be a strong risk factor for cancer development [11]; Hp a-carbonic anhydrase inhibitors may be introduced in the management of gastric ulcers and cancer [12]; pancreatic CAll-expressing cells give rise to new islet and acinar cells following birth and injury which may arise pancreatic malignancy from the uncontrolled growth of progenitor/stem cells [13]; bone marrow-derived stem cells (BMDSCs) are involved in the development and/or progression of pancreatic cancer [14]; and $\mathrm{Hp}$-I by recruiting BMDSCs may facilitate cancer development and progression in animal models and humans $[15,16]$.

Therefore, $H p$ eradication may also positive influence AIP patients by ameliorating the autoimmune sequelae and subsequent development of pancreatic cancer, and thus further studies are needed.

\section{References}

1. Li, P. et al. (2020) The potential role of bacteria in pancreatic cancer: A systematic review. Carcinogenesis, 2020; doi: 10.1093/carcin/bgaa013.

2. Shinji, A., et al. (2004) Autoimmune pancreatitis is closely associated with gastric ulcer presenting with abundant IgG4-bearing plasmacell infiltration. Gastrointest. Endosc., 59, 506-511. 
3. Kountouras, J., et al. (2005) A concept on the role of Helicobacter pylori infection in autoimmune pancreatitis. Cell. Mol. Med., 9, 196-207.

4. Diamantidis, M., et al. (2008) New aspects of regulatory signaling pathways and novel therapies in pancreatic cancer. Curr. Mol. Med., 8, 1237.

5. Kountouras, J., et al. (2005) Apoptotic and Anti-Angiogenic Strategies in Liver and Gastrointestinal Malignancies. J. Surg. Oncol., 90, 249-259.

6. Frulloni, L., et al. (2009) Identification of a Novel Antibody Associated with Autoimmune Pancreatitis. N. Engl. J. Med., 361, 2135-2142.

7. Jönsson, K., et al. (2004) Molecular cloning and characterization of two Helicobacter pylori genes coding for plasminogen-binding proteins. Proc. Natl. Acad. Sci. U. S. A., 101, 1852-1857.

8. Kountouras, J., et. al. (2007) Challenge in the pathogenesis of autoimmune pancreatitis: potential role of Helicobacter pylori infection via molecular mimicry. Gastroenterology 133, 368-369.

9. Guarneri, F., et al. (2005) Helicobacter pylori and autoimmune pancreatitis: role of carbonic anhydrase via molecular mimicry J. Cell. Mol, Med., 9, 741-744.

10. Hirano, K., et a. (2014) Association between autoimmune pancreatitis and malignancy. Clin. J. Gastroenterol., 7, 200-204.

11. Asano,T., et al. (2015) Association Between Immunoglobulin G4-related Disease and Malignancy within 12 Years after Diagnosis: An Analysis after Longterm Followup. J. Rheumatol., 42, 2135-2142.

12. Nishimori, I., et al. (2006) Carbonic Anhydrase Inhibitors: DNA Cloning and Inhibition Studies of the a-Carbonic Anhydrase from Helicobacter 
pylori, A New Target for Developing Sulfonamide and Sulfamate Gastric Drugs. J. Med. Chem., 49, 2117-2126

13. Inada, A., et al. (2008). Carbonic anhydrase II-positive pancreatic cells are progenitors for both endocrine and exocrine pancreas after birth. Proc. Natl. Acad. Sci. U. S. A., 105, 19915-19919.

14. Blogowski, W., et al. (2016) Concise Review: Pancreatic Cancer and Bone Marrow-Derived Stem Cells. Stem Cells Transl. Med., 5, 938-945.

15. Kountouras, J., et al. (2015) Bone Marrow-Derived Stem Cells in Pathogenesis of Helicobacter pylori-Associated Gastrointestinal Cancer. Clin. Transl. Gastroenterol., 6, e129.

16. Kountouras, J., et al. (2017). Active Helicobacter pylori Infection Is a Risk Factor for Colorectal Mucosa: Early and Advanced Colonic Neoplasm Sequence. Gut Liver., 15, 733-734. 